\title{
Hypoxia during one lung ventilation in thoracic surgery
}

Gordana Taleska,MD,MSc, Andriy Grynyuk,MD, Maja Sostaric, MD,PhD

University Medical Centre, Department of anesthesia and perioperative intensive therapy

Ljubljana, Slovenia

Background and Goal of Study: The technique of one lung ventilation (OLV) is instituted with the purpose to achieve isolation of the diseased lung being operated upon, using double-lumen endobronchial tube. Thoracic surgical procedures which are performed in lateral decubitus position nowadays couldn't be imagined without OLV. In spite advantages regarding surgical exposure, OLV is associated with serious respiratory impairment. Hypoxemia is considered to be the most important challenge during OLV. The goal of this study was to establish the magnitude of intrapulmonary shunt, as well as the immensity of hypoxia during general anesthesia with OLV.

Materials and Methods: In this prospective interventional clinical study we enrolled thirty patients who underwent elective thoracic surgery with prolonged period of OLV. The patients received balanced general anesthesia with fentanyl/propofol/rocuronium. A double-lumen endobronchial tube was inserted in all patients, and mechanical ventilation with $50 \%$ oxygen in air was used during the entire study. Arterial blood gases were recorded in a lateral decubitus position with two-lung ventilation, at the beginning of OLV (OLV 0) and 10 and $30 \mathrm{~min}$. (OLV 10, OLV 30, respectively) after initiating OLV in all patients. The monitoring was standard. Arterial partial pressure of oxygen $\left(\mathrm{PaO}_{2}\right)$, arterial oxygen saturation $\left(\mathrm{SaO}_{2}\right)$ and venous admixture percentage - intrapulmonary shunt (Qs/Qt \%) were measured, as well as mean arterial pressure and heart rate during the same time intervals. For the purpose of this study, the quantitative value of $\mathrm{Qs} / \mathrm{Qt} \%$ was mathematically calculated by the blood gas analyzer AVL Compact 3. A p value $<0.05$ was taken to be statistically significant.
Results: When OLV was instituted arterial oxygenation decreased, whereas the shunt increased, about $10 \mathrm{~min}$. of the commencement, with improvement of the oxygenation approximately half an hour afterwards. Statistically relevant difference $(p<0,05)$ occurred regarding $\mathrm{PaO}_{2}, \mathrm{SaO}_{2}$ and Qs/Qt in the different measurings.
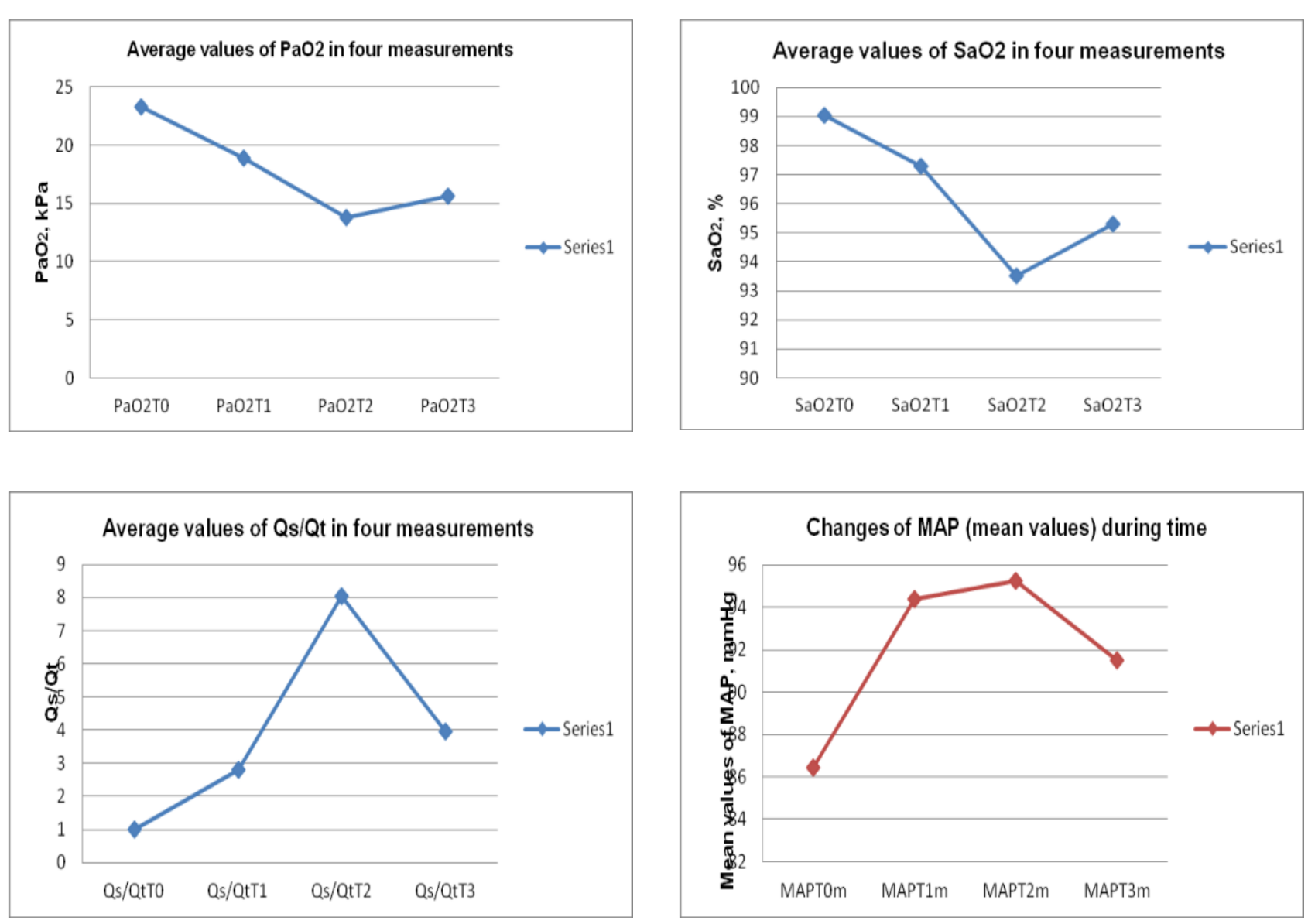

Conclusions: Hypoxia during OLV with increase of intrapulmonary shunt usually occurs after $10 \mathrm{~min}$. of its initiation. Following $30 \mathrm{~min}$. of the beginning of OLV, the values of the shunt regularly decrease back to the normal quantities. 